\title{
AN ISOMORPHISM THEOREM FOR VALUATED VECTOR SPACES
}

\author{
PAUL HILL ${ }^{1}$
}

\begin{abstract}
The following isomorphism theorem is proved for valuated vector spaces. Let $N$ and $N^{\prime}$ be nice subspaces of free valuated vector spaces $F$ and $F^{\prime}$, respectively. If $N$ and $N^{\prime}$ have isomorphic basic subspaces and if the quotient spaces $F / N$ and $F^{\prime} / N^{\prime}$ are isomorphic, there exists an isomorphism from $F$ onto $F^{\prime}$ that maps $N$ onto $N^{\prime}$ and induces the given isomorphism on the quotient spaces. In particular, $N$ and $N^{\prime}$ are isomorphic.
\end{abstract}

Throughout let $\Gamma$ denote a fixed totally ordered set with suprema; every nonempty subset of $\Gamma$ has a least upper bound in $\Gamma$. Adjoin to $\Gamma$ an element $\infty$ that is considered larger than every element of $\Gamma$. Following [2] and [4], we define a valuated vector space to be a vector space $V$ with values in $\{\Gamma, \infty\}$ satisfying the following conditions:

(1) $|x+y| \geqslant \min \{|x|,|y|\}$ for all $x, y \in V$.

(2) $|c x|=|x|$ if $c$ is a nonzero scalar.

(3) $|x|=\infty$ if and only if $x=0$.

We shall refer to a valuated vector space simply as a valuated space. If $V$ and $W$ are valuated spaces, a map from $V$ to $W$ is a linear transformation that does not decrease values. Products and coproducts exist [2], and the coproduct of valuated spaces $\left\{V_{i}\right\}_{i \in I}$ is nothing more than the direct sum $\Sigma_{i \in I} \oplus V_{i}$ where the value of $x=\sum x_{i}$ with $x_{i} \in V_{i}$ is given by $|x|=\min \left\{\left|x_{i}\right|\right\}$.

The structure of valuated vector spaces is much more complicated than the structure of (nonvaluated) vector spaces. A fundamental feature of vector spaces that is missing in valuated spaces is the existence of a basis for every space. Naturally, a valuated space $V$ has a basis $\left\{x_{i}\right\}$ when $V$ is the coproduct of the one-dimensional spaces $\left\langle x_{i}\right\rangle$; in such case, $V$ is said to be free. Thus having a basis and being free are equivalent. Every countably generated valuated space has a basis [1]. But in the uncountable case, even a subspace of a space with a basis need not have a basis; the author gave a necessary and sufficient condition for a subspace of a free valuated space to be again free in [3]. Although not every valuated space has a basis, it is true that every valuated space $V$ has a basic subspace $B$. By this we mean that $B$ has a basis and, for each nonzero $x \in V$, there exists $b \in B$ such that $|x-b|>|x|$. The latter property, in some sense, makes $B$ dense in $V$; see Theorem 4 in [2].

Received by the editors August 10, 1982.

1980 Mathematics Subject Classification. Primary 18B99, 18E05; Secondary 18G05, 20 K99.

Key words and phrases. Valuated vector space, basis, nice subspace of free space (NSF-space), isomorphism, uniqueness theorem.

'Sponsored by NSF Grant MCS 8102470 . 
If $W$ is a subspace of the valuated space $V$, the valuation on the quotient space is given by

$$
|x+W|=\sup \{|x+w|: w \in W\},
$$

and the subspace $W$ is said to be a nice subspace of $V$ if for every $x$ the supremum is obtained, that is, if $|x+W|=|x+w|$ for some $w \in W$. It was once thought that if $V$ had a basis then every nice subspace $W$ of $V$ also would have a basis, but it turned out differently [5] (see also Theorem 6.3 in [4]). Following [3] and [4], we call a nice subspace of a free space an NSF-space. Equivalently, an NSF-space is a nice subspace of a valuated vector space that has a basis. Although NSF-spaces are not always free, we are able to prove here a structural uniqueness theorem for NSF-spaces. Our main result is the following isomorphism theorem.

THEOREM. Let $N$ and $N^{\prime}$ be nice subspaces of free valuated vector spaces $F$ and $F^{\prime}$, respectively. If $N$ and $N^{\prime}$ have isomorphic basic subspaces and if the quotient spaces $F / N^{\circ}$ and $F^{\prime} / N^{\prime}$ are isomorphic (all in the category of valuated spaces), there exists an isomorphism from $F$ onto $F^{\prime}$ that takes $N$ onto $N^{\prime}$ and induces the given isomorphism on the quotient spaces. In particular, $N$ and $N^{\prime}$ are isomorphic.

Proof. Let $\sigma: B \longrightarrow B^{\prime}$ be an isomorphism between basic subspaces of $N$ and $N^{\prime}$, respectively, and let $\phi: F / N \longrightarrow F^{\prime} / N^{\prime}$ be an isomorphism between the quotient spaces. First, observe that if $C / N$ is a basic subspace of $F / N$ then $C=N \oplus A$ is the coproduct of $N$ and a free subspace $A$ of $F$. We use $\oplus$ throughout for coproduct. Moreover, $B \oplus A$ is a basic subspace of $F$ since $B$ is basic in $N$ and $C / N \cong A$ is basic in $F / N$. Likewise, $B^{\prime} \oplus A^{\prime}$ is basic in $F^{\prime}$ if $A^{\prime}$ is chosen similarly to $A$. But $A \cong C / N$ and $A^{\prime} \cong C^{\prime} / N^{\prime}$ are isomorphic because they are basic subspaces of isomorphic spaces (Theorem 4 in [2]). By hypothesis, $B \cong B^{\prime}$. Therefore $A \oplus B \cong A^{\prime}$ $\oplus B^{\prime}$, which implies that

$$
F \cong A \oplus B \cong A^{\prime} \oplus B^{\prime} \cong F^{\prime}
$$

since $F$ and $F^{\prime}$ are basic subspaces of themselves. Thus we can write $F=\Sigma_{i \in I} \oplus\left\langle x_{i}\right\rangle$ and $F^{\prime}=\sum_{i \in I} \oplus\left\langle y_{i}\right\rangle$, where $\left|x_{i}\right|=\left|y_{i}\right|$ for each $i$. If $J \subseteq I$, define $F_{J}=\sum_{j \in J} \oplus\left\langle x_{j}\right\rangle$ and $F_{J}^{\prime}=\Sigma_{j \in J} \oplus\left\langle y_{j}\right\rangle$.

Suppose that for some subset $J$ of $I$ that $M$ and $M^{\prime}$ are subspaces of $F$ and $F^{\prime}$ containing $F_{J}$ and $F_{J}^{\prime}$, respectively, such that $M / F_{J}$ and $M^{\prime} / F_{J}^{\prime}$ are finitely generated. Further, assume that $\pi: M \longrightarrow M^{\prime}$ is an isomorphism satisfying the following conditions:

(1) $\pi$ maps $F_{J}$ onto $F_{J}^{\prime}$.

(2) $\pi(a)+N^{\prime}=\phi(a+N)$ if $a \in M$.

(3) $|b+a|>|b| \Leftrightarrow|\sigma(b)+\pi(a)|>|\sigma(b)|$ whenever $b \in B$ and $a \in M$.

Observe that if $M=F$ then $M^{\prime}=F^{\prime}$ since $M^{\prime} / F_{J}^{\prime} \cong M / F_{J}$ and $M$ is finitely generated over $F$, and in this case the conclusion of the theorem follows from condition (2). Thus our aim is to enlarge the domain of $\pi$ (until we obtain $M=F$ ). Suppose that $x \in F$ but $x \notin M$. Since $M / F_{J}$ is finitely generated and since $F_{J}$ is a direct summand (and consequently a nice subspace) of $F, M$ must be a nice subspace 
of $F$; see Lemma 6 in [2]. Likewise, $M^{\prime}$ is a nice subspace of $F^{\prime}$. It follows that $\langle x, M\rangle=\langle x+m\rangle \oplus M$ for some $m \in M$, and, by replacing $x$ by $x+m$, we may assume that $\langle x, M\rangle=\langle x\rangle \oplus M$. We seek $y \in F^{\prime}$ such that $|x|=|y|,\left\langle y, M^{\prime}\right\rangle=$ $\langle y\rangle \oplus M^{\prime}$ and $\phi(x+N)=y+N^{\prime}$. Two cases need to be distinguished. Even though $N$ is nice, the first case cannot be eliminated because $x$ has already been chosen orthogonal to $M$.

Case 1. $|x+N|>|x|$. Let $\phi(x+N)=w+N^{\prime}$ where the representative $w$ is chosen so that $|w|>|x|$. Choose $n \in N$ so that $|x+n|>|x|$, and choose $b \in B$ so that $|n-b|>|n|=|x|$. Observe that $|x+b|>|x|=|b|$. Define $b^{\prime}=\sigma(b)$ and set $y=w-b^{\prime}$. Since $|w|>|x|$ and $\left|b^{\prime}\right|=|b|=|x|$, we know that $|y|=|x|$, and $\phi(x+N)=w+N^{\prime}=y+N^{\prime}$ since $b^{\prime} \in N^{\prime}$. In order to show that $\left\langle y, M^{\prime}\right\rangle=$ $\langle y\rangle \oplus M^{\prime}$, assume the contrary and suppose that $\left|y+m^{\prime}\right|>|y|$ for some $m^{\prime} \in M^{\prime}$. This immediately yields $\left|m^{\prime}-b^{\prime}\right|>|y|=\left|b^{\prime}\right|$. Condition (3) implies that $|m-b|$ $>|b|$, where $\pi(m)=m^{\prime}$. But this leads to $|x+m|>|x|$ because $|x+b|>|x|$ $=|b|$. However, $|x+m|>|x|$ is impossible because $x$ is orthogonal to $M$. Thus we conclude that $\left\langle y, M^{\prime}\right\rangle=\langle y\rangle \oplus M^{\prime}$. The mapping $\pi$ is extended by mapping $x$ onto $y$. Furthermore, condition (1) is automatically retained by the extension, and we have chosen $y$ so that condition (2) is retained. We need to verify that condition (3) survives. Toward that end, let $c$ denote an arbitrary element of $B$; recall that $b$ now represents a special element of $B$. Let $c^{\prime}=\sigma(c)$, choose $m \in M$ and let $m^{\prime}=\pi(m)$. We need only verify that $|x+m+c|>|c|$ precisely when $\left|y+m^{\prime}+c^{\prime}\right|>\left|c^{\prime}\right|$. Since $c^{\prime}=\sigma(c),|c|=\left|c^{\prime}\right|$; denote the common value by $\lambda$. Notice that the case that $\lambda>|x|$ precludes $|x+m+c|>|c|$ because $|x+m| \leqslant|x|$. Likewise, $\mid y+m^{\prime}+$ $c^{\prime}|>| c^{\prime} \mid$ is precluded in this case. Thus we may assume that $\lambda \leqslant|x|$. If $\lambda<|x|$, $|x+m+c|>|c|$ exactly when $|m+c|>|c|$. But $|m+c|>|c|$ if and only if $\left|m^{\prime}+c^{\prime}\right|>\left|c^{\prime}\right|$, and the latter is true exactly when $\left|y+m^{\prime}+c^{\prime}\right|>\left|c^{\prime}\right|$ in case $\lambda<|x|$. Therefore, we may assume that $\lambda=|x|$. In this case, $|x+m+c|>\lambda$ is equivalent to $|m+c-b|>\lambda$ and $\left|y+m^{\prime}+c^{\prime}\right|>\lambda$ is equivalent to $\left|m^{\prime}+c^{\prime}-b^{\prime}\right|$ $>\lambda$. But $|m+c-b|>\lambda$ and $\left|m^{\prime}+c^{\prime}-b^{\prime}\right|>\lambda$ fall or stand together because if either is valid then $\lambda=|c-b|=\left|c^{\prime}-b^{\prime}\right|$ unless $|m|>\lambda$. Moreover, the case $|m|>\lambda$ is trivial. This completes the verification that (3) remains valid for the extension of $\pi$ to $\langle x\rangle \oplus M$. Now, we show that $\pi$ can also be successfully extended in the alternate case.

Case 2. $|x+N|=|x|$. If $|x+m+N|>|x|$ for some $m \in M$ where $|m|=|x|$ then because $x+m$ is also orthogonal to $M$ we can return to Case 1 by replacing $x$ by $x+m$. Therefore, we may assume that if $m \in M$ and $|m|=|x|$ it is the case that $|x+m+N|=|x|$. Now, let $\phi(x+N)=y+N^{\prime}$ where $|y|=|x|$. Suppose that $\left|y+m^{\prime}\right|>|y|$ for some $m^{\prime} \in M^{\prime}$ and let $\pi(m)=m^{\prime}$. It follows at once that $\phi(x+m+N)=y+m^{\prime}+N^{\prime}$ since $\phi(m+N)=m^{\prime}+N^{\prime}$. Thus $|x+m+N|>$ $|x|$, but this has already been ruled out since $|m|=\left|m^{\prime}\right|=|y|=|x|$. Therefore, $\left|y+m^{\prime}\right| \leqslant|y|$ for each $m^{\prime} \in M^{\prime}$, and $\left\langle y, M^{\prime}\right\rangle=\langle y\rangle \oplus M^{\prime}$. Again, we extend $\pi$ by mapping $x$ onto $y$. The verification of conditions (1)-(3) for the extended map is similar to Case 1 except that $|x+m+c|>|c|=|x|$ for $c \in B$ does not occur, nor does $\left|y+m^{\prime}+c^{\prime}\right|>\left|c^{\prime}\right|=|y|$, under the present hypothesis. 
Due to the symmetry between $F$ and $F^{\prime}$ it follows from the above local extension of $\pi$ that $\pi: M \gg M^{\prime}$ satisfying conditions (1)-(3) can always be extended, by a sequence of local extensions of $\pi$ and $\pi^{-1}$, to an isomorphism $F_{K} \rightarrow F_{K}^{\prime}$ that still satisfies conditions (1)-(3), where $K$ is a countable extension of the set $J$ in $I$. Thus if $J$ is chosen maximal then $J=I$, and the theorem is proved.

COROLlaRY 1. If $N$ and $N^{\prime}$ are isomorphic nice subspaces of a free valuated vector space $F$ with isomorphic quotients $F / N$ and $F / N^{\prime}$, then $N$ and $N^{\prime}$ are equivalent subspaces of $F$ in the sense that there is an automorphism of $F$ that maps $N$ onto $N^{\prime}$.

COROLlaRY 2. Let $N$ be a nice subspace of a free valuated space $F$. If the structure of the valuated space $V=F / N$ can be determined by certain cardinal numbers, then the same is true of $N$.

Proof. In view of the theorem, the space $N$ is determined up to isomorphism by a basic subspace $B$ of $N$ together with $V=F / N$.

The basic subspace $B$ is determined by cardinal numbers since it is free.

REMARK. The preceding corollary is stated somewhat loosely. But when we say that a valuated vector space $V$ is determined by certain cardinal numbers what we actually mean is that $V$ is determined, up to isomorphism, by a function or a sequence of functions from $\Gamma$ to the cardinal numbers.

For our next result we specialize to the case that $\Gamma$ is well ordered although this does not yield the most general result. Let $V=\Pi_{\gamma \in \Gamma} H_{\gamma}$ be the product of homogeneous spaces $H_{\gamma}$ of value $\gamma$, which means that the value of each nonzero element of $H_{\gamma}$ is $\gamma$. Among such products, $V$ is determined by the cardinal-valued function $f(\gamma)=\operatorname{dim}\left(H_{\gamma}\right)$. This observation leads to the following result; it is of interest only in the case that $N$ does not have a basis.

COROLlaRY 3. Suppose that $\Gamma$ is well ordered and that $F / N=\prod_{\gamma \in \Gamma} H_{\gamma}$, where $H_{\gamma}$ is homogeneous of value $\gamma$. As before, assume that $N$ is a nice subspace of the free valuated space $F$. Up to isomorphism, $N$ is uniquely determined by the cardinal-valued functions $f(\gamma)=\operatorname{dim}\left(H_{\gamma}\right)$ and $g(\gamma)=\operatorname{dim}\left(B_{\gamma}\right)$, where $\Sigma_{\gamma \in \Gamma} \oplus B_{\gamma}$ is a basic subspace of $N$ with $B_{\gamma}$ being homogeneous of value $\gamma$.

\section{REFERENCES}

1. R. Brown, Valued vector spaces of countable dimension, Publ. Math. Debrecen 18 (1971), 149-151.

2. L. Fuchs, Vector spaces with valuations, J. Algebra 35 (1975), 23-38.

3. P. Hill, Criteria for freeness in groups and valuated vector spaces, Lecture Notes in Math., vol. 616. Springer-Verlag, Berlin and New York, 1977, pp. 140-157.

4. P. Hill and E. White, The projective dimension of valuated vector spaces, J. Algebra 74 (1982). 374-401.

5. F. Richman and E. Walker, Valuated groups, J. Algebra 56 (1979), 145-167.

Department of Mathematics, Auburn University, Auburn, Alabama 36849 